Indian J. Phys. 65B (6), 595-599 (1991)

\title{
An adaptive response of Vibrio cholerae strain OGAWA 154 to furazolidone
}

\author{
Radha Bhattacharya \\ Biophysics Division, Saha Institute of Nuclear Physics, \\ 37/1, Belgachia Road, Cafcutta-700 037, India
}

\begin{abstract}
Furazolidone is a synthetic drug of nitrofuran group which display antimicrobial activity, hence an adaptive response of this particular drug to microorganism seems to be very important. This response was studied in Vibrio cholerae system. The study showed that cells adapted with low concentration of furazolidone were one hundred per cent resistant to subsequent challenging dose in comparison to non-adapted cells. Further adapted cells showed better survival subjected to different challenging doses.
\end{abstract}

Keywords : Vibrio cholerae, adaptive response, nitrofuran group of drugs.

PACS No: 87.22.-q

\section{Introduction}

Furazolidone is an antimicrobial drug (Paul and Paul 1964). It has a significant application in cholera therapy. In this respect an adaptive response of this drug to cholera bacteria appears to be very important and useful. This study was carried out with Vibrio cholerae cells. With a view to establish this response, a dose-response relation to these cells to furazolidone and the kinetics of inactivation of the drug were studied. An adaptive response study were made by the log phase cells of $V$. cholerae treated with low dose of drug for various times and then the treated cells were subjected to a challenging dose. Earlier reports of Samson and Cairns (1977) indicated that if $E$. coli cells were first exposed to low concentrations of the methylating agent $N$-methyl- $N$-nitro- $N$-nitrosoguanidine (MNNG), they became resistant to the mutagenic and lethal effects of a subsequent challenge with a higher dose of this agent. This response is termed an adaptive response (Jeggo et al 1977), it is inducible and carries out some form of DNA repair system as described by Witkin (1967) and George et al (1974). Further furazolidone was shown to preferentially inhibit DNA synthesis in V. choleroe cells (Raichaudhuri et al 1970, Chatterjee and Raichowdhuri 1971) and induce prophage $\lambda$ in $E$. coli (Pal and Chatterjee 1985). Hence an adaptive response Istudy in V. cholerae cells with furazolidone appears to be interesting. 